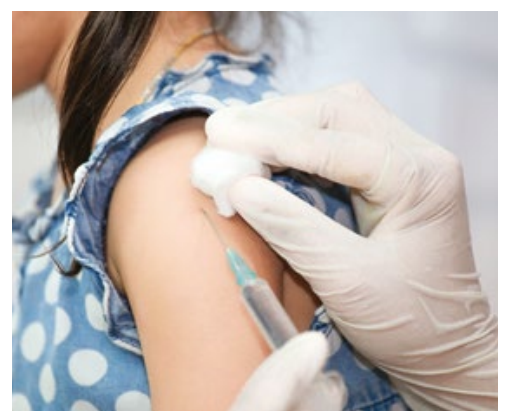

CPD

\section{Julia ML Brotherton}

\section{Background}

Australia has included quadrivalent human papillomavirus (HPV)

vaccination in its national program since 2007. Significant declines have been observed in the incidence of HPV infection, genital warts and high-grade cervical lesions. In 2018, the program changed to a nonavalent HPV vaccine administered over a routine two-dose schedule for the target cohort of adolescents aged 12-13 years.

\section{Objectives}

The aim of this article is to provide an overview of the nonavalent HPV vaccine, the rationale for its use and expected outcomes, and to review the updated dose scheduling requirements for HPV vaccines.

\section{Discussion}

The nonavalent HPV vaccine will broaden the impact of HPV vaccination, primarily against cervical cancer and pre-cancer. A two-dose schedule with an interval of 6-12 months between doses is appropriate for those aged $\leq 14$ years at the time of first dose. Older individuals and those who are immunocompromised should continue to receive the three-dose schedule at zero, two and six months.

\title{
Human papillomavirus vaccination update
}

\author{
Nonavalent vaccine and the two-dose schedule
}

THIS YEAR, 2018, is the 12th year of human papillomavirus (HPV) vaccine delivery as part of the National Immunisation Program in Australia. Australia's 'big bang' approach, with a large-scale catch-up program using the quadrivalent HPV vaccine (trade name Gardasil) initially offered to all females aged 12-26 years, has since been shown to be highly effective. Males have also been offered vaccination since 2013, with an initial catch-up program to age 15 years. Protection is believed to be antibodymediated, with vaccine-generated antibodies at very low levels able to block a conformational change the virus needs to make in order to enter the basal epithelial cell. ${ }^{1}$ Substantial declines in the incidence of genital warts (caused by HPV 6 and 11), ${ }^{2}$ vaccine-targeted HPV infections $(6,11,16 \text { and } 18)^{3}$ and cervical pre-cancers ${ }^{4}$ have been documented, with linked registry data showing vaccine effectiveness. ${ }^{5}$ Recently, a decrease in new cases of juvenile-onset recurrent respiratory papillomatosis, a disease caused by vertical transmission of HPV 6 and 11 , has also been observed. ${ }^{6}$

High, and recently increasing, HPV vaccine coverage in the routinely vaccinated cohorts (age 12-13 years, first year of high school in most states and territories) indicates the acceptability of the school-based immunisation approach. Vaccine coverage from the three doses respectively at age $14-15$ years in 2016 is $87 / 85 / 79 \%$ in females and $83 / 80 / 74 \%$ in males. ${ }^{7}$ General practice plays an important part in HPV vaccination in
Australia, specifically in immunising those who do not wish to, or cannot, be immunised at school and in catching up those who missed doses in the school program, with catch-up now funded up to the age of 19 years. Provider endorsement is known to be a powerful motivator for $\mathrm{HPV}$ vaccination, as for other vaccines. In this article, we bring general practitioners (GPs) up to date with the evidence supporting the use of the nonavalent HPV vaccine (trade name Gardasil 9) and the two-dose schedule for HPV vaccines.

\section{Nonavalent HPV vaccine: Purpose}

The nonavalent HPV vaccine was developed to extend the protection offered by the vaccine from HPV 6 and 11, which cause genital warts, and HPV 16 and 18, which are the most oncogenic types, to include the next five most frequently detected oncogenic HPV types in cervical cancers (Table 1). HPV 16 is the most oncogenic HPV type, predominating across all HPV-related cancer sites (approximately $61 \%$ cervical [higher proportion at younger ages], $81 \%$ anal, $59 \%$ vaginal, $68 \%$ vulval, $69 \%$ penile and $82 \%$ oropharyngeal HPV-related cancers). ${ }^{8-11}$ Thus, maintenance of strong protection against HPV 16 is of utmost importance when extending vaccine protection to other types. HPV 18 is the second most common type across all cancers but is as frequent as HPV 16 in cervical adenocarcinomas. ${ }^{12}$ The additional five types added (HPV 31, 33, $45,52,58)$ are each responsible for a few 
per cent of cervical cancers; cumulatively, however, the overall protection against cervical cancer afforded by protection against HPV 16,18, 31, 33, 45, 52 and 58 is estimated at $89 \%$ globally. ${ }^{13} \mathrm{~A}$ recent Australian study of 847 cervical cancers estimated the protection in Australia may be as high as $93 \%$ (77\% HPV 16, 18 and $16 \%$ HPV 31, 33, 45, 52, 58). ${ }^{14}$

\section{Composition}

The nonavalent vaccine is manufactured similarly to the quadrivalent vaccine. It contains virus-like particles (VLPs) for HPV types $6,11,16,18,31,33,45,52$ and 58 , with an increased amount for types 16 , 18 and 6, compared with the quadrivalent vaccine. The VLPs consist of the HPV outer coat protein L1 and self-assemble into particles following recombinant expression in a yeast vector system. The vaccine uses the same aluminium adjuvant as the quadrivalent vaccine but in a greater amount. The increase in VLP and aluminium concentration is to ensure the maintenance of effective protection against the quadrivalent HPV types while increasing protection to include the five additional types. ${ }^{15}$

\section{Efficacy and immunogenicity}

A randomised controlled trial of over 14,000 women aged $16-26$ years in 18 countries found that three doses of the nonavalent HPV vaccine (at zero, two and six months) provided $97 \%$ efficacy against HPV 31/33/45/52/58-related high-grade cervical, vulvar and vaginal intraepithelial neoplasia when administered to women not previously infected with those types. ${ }^{16}$ The comparator group in the study received the quadrivalent vaccine - given its proven efficacy, use of a placebo would have been unethical. Protection against HPV 6, 11, 16 and 18 was confirmed by demonstration that the antibody titres generated by the two vaccines were equivalent.

Immunobridging from females to males aged 16-26 years has been established in two studies. Both found equivalent antibody titres in heterosexual males. ${ }^{17,18}$ Among 300 men who have sex with men (MSM), although seroconversion rates remained over $99 \%$, antibody titres were noted to be lower. ${ }^{18}$ The clinical significance of this, if any, is not known.

\section{Two-dose schedules}

The surprisingly strong immunogenicity of HPV vaccines, which are non-live, subunit vaccines, led to evaluation of effectiveness when administered in only two doses (ie a more widely spaced prime-boost effect rather than a primeprime-boost pattern. ${ }^{1}$ Studies have shown that the immunogenicity of the HPV vaccines, when administered using two doses spaced 6-12 months apart to adolescents aged $\leq 14$ years at first dose, is equivalent to that achieved using three doses of vaccine in adult women. ${ }^{19}$ In 2014, the World Health Organization (WHO) recommended the routine use of two-dose HPV vaccine schedules for younger adolescents, but those aged $\geq 15$ years and those who are immunocompromised still require three doses. ${ }^{20}$ Randomised trials are currently underway to assess whether even one dose of HPV vaccine may be effective.

The nonavalent HPV vaccine has been evaluated in a trial comparing antibody responses in adolescents aged 9-14 years receiving two doses six or 12 months apart with responses seen in women aged 16-26 years receiving three doses. ${ }^{21}$

\section{Table 1. Comparison of human papillomavirus vaccines}

\begin{tabular}{|c|c|c|c|}
\hline & Bivalent HPV vaccine (Cervarix) & $\begin{array}{l}\text { Quadrivalent HPV vaccine } \\
\text { (Gardasil) }\end{array}$ & $\begin{array}{l}\text { Nonavalent HPV vaccine } \\
\text { (Gardasil 9) }\end{array}$ \\
\hline L1 virus-like particle types & HPV 16, 18 & HPV 6, 11, 16, 18 & HPV 6, 11, 16, 18, 31, 33, 45, 52, 58 \\
\hline Adjuvant & $\begin{array}{l}\text { ASO4 (0.5 mg aluminium } \\
\text { hydroxide and } 50 \mu \mathrm{g} \\
\text { 3-O-desacyl-4"--monophosphoryl } \\
\text { lipid A [MPL]) }\end{array}$ & $\begin{array}{l}0.225 \mathrm{mg} \text { aluminium } \\
\text { hydroxyphosphate sulphate }\end{array}$ & $\begin{array}{l}0.5 \mathrm{mg} \text { aluminium } \\
\text { hydroxyphosphate sulphate }\end{array}$ \\
\hline Expression system & Baculovirus-insect cell & Yeast & Yeast \\
\hline Cross-protection & High against HPV 31, 33, 4535,36 & Limited; some against HPV $31^{37}$ & Nil known \\
\hline Registered for use in males & No & Yes & Yes \\
\hline Schedule & \multicolumn{3}{|c|}{$\begin{array}{l}\text { Two doses spaced 6-12 months apart for those aged } 14 \text { years and under at first dose } \\
\text { Three doses spaced at zero, two and six months for those aged } 15 \text { years and over at first dose and } \\
\text { immunocompromised individuals with select major medical conditions* }\end{array}$} \\
\hline \multicolumn{4}{|c|}{$\begin{array}{l}\text { * Those who should receive a three-dose schedule include individuals with the following major medical conditions: primary or secondary immunodeficiencies } \\
\text { (B lymphocyte antibody and T lymphocyte complete or partial deficiencies), human immunodeficiency virus infection, malignancy, organ transplantation or } \\
\text { significant immunosuppressive therapy (excluding asplenia or hyposplenia). The recommendation for a three-dose schedule does not apply to children aged } \\
\leq 14 \text { years with asplenia, asthma, chronic granulomatous disease, chronic heart/liver/lung/renal disease, central nervous system anatomic barrier defects } \\
\text { (eg cochlear implant), complement deficiency, diabetes or sickle cell disease, in the absence of any of the above conditions. } \\
\text { HPV, human papillomavirus }\end{array}$} \\
\hline
\end{tabular}


Antibody titres to all nine types were non-inferior (and were, in fact, higher at younger ages).

\section{HPV vaccine safety}

Since 2006, HPV vaccines have been used globally in over 270 million doses and scrutinised by routine surveillance systems and high-quality population-based studies assessing potential vaccine safety signals. There is no evidence that any serious illness occurs more commonly in vaccinated than unvaccinated individuals, including autoimmune and neurological conditions. ${ }^{22}$ The WHO Global Advisory Committee on Vaccine Safety has reviewed the vaccines seven times and continues to find them safe..$^{23}$

Trial data indicate a higher rate of injection site reactions (any pain, swelling, redness) in young women receiving the nonavalent vaccine than in those receiving the quadrivalent vaccine ( $91 \%$ vs $85 \%$ ). This may be attributable to the increased amount of adjuvant. ${ }^{24}$ There was no difference in rates of systemic or serious adverse events. Rates of injection site reactions to the nonavalent vaccine are lower in males and in adolescents aged 9-14 years than in adult women. ${ }^{17}$

\section{Australian program}

In 2018, the nonavalent HPV vaccine, in two doses spaced 6-12 months apart for the routine school age cohort (usual age 12-13 years), is the vaccine and schedule in use in the national program. ${ }^{25}$ School-based immunisation programs operate in every state and territory and require parental consent to vaccinate the child at school. In some jurisdictions, GPs are routinely involved in catching up doses missed in the school program. All GPs may be involved in providing HPV vaccine to those with special needs, immunocompromise or vaccine hesitancy, or as catch up to those aged up to 19 years, now that HPV vaccine availability for catch up has been extended (since July 2017). GPs may also proactively discuss elective HPV vaccination in those who are not funded under the national program but in whom vaccination is recommended or may be of particular benefit.

\section{Higher risk groups}

$\mathrm{HPV}$ vaccine is recommended but not funded for immunocompromised individuals and for MSM. ${ }^{25}$ No age limit is set on these recommendations. MSM have a markedly elevated risk of anal cancer and HPV infection, and re-infection with HPV is common. ${ }^{26}$ The epidemiology of HPV infection is different in males and females, with HPV infection rates in males consistent throughout life, probably because of a low likelihood of males generating protective immunity through natural infection. ${ }^{27}$

The vaccine may be of benefit to prevent re-infection and spread in the anal canal regardless of previous exposure. The three-dose vaccine is also recommended for immunocompromised individuals, who may be at higher risk of HPV-related disease and cancers. Immunocompromised individuals include those with the following major medical conditions: primary or secondary immunodeficiencies (B lymphocyte antibody and $\mathrm{T}$ lymphocyte complete or partial deficiencies), human immunodeficiency virus infection, malignancy, organ transplantation or significant immunosuppressive therapy (but not asplenia or hyposplenia)..$^{25,28}$ The recommendation for a three-dose schedule does not apply to children aged $\leq 14$ years with asplenia, asthma, chronic granulomatous disease, chronic heart, liver, lung or renal disease or central nervous system anatomical barrier defects (eg cochlear implant), complement deficiency, diabetes or sickle cell disease, in the absence of any of the above conditions. ${ }^{28}$

The vaccine should also be considered for women who have undergone treatment for cervical intraepithelial neoplasia. Women in this group have shown an inability to clear HPV infection and have a long-term elevated risk of cervical cancer. ${ }^{29}$ While the vaccine cannot treat existing infection or disease, there is some evidence that, by stimulating protective levels of antibodies, it may prevent re-infection and subsequent disease with either the same type of HPV that caused the lesion or another type. ${ }^{30}$

Women with a single positive cervical screening test (ie positive for HPV 16 and 18 or other oncogenic HPV types) have a current HPV infection that may be either an acute infection or a persisting infection. Vaccination will not alter the course of the current infection but, as for any woman, could prevent future infections with vaccine-covered HPV types. A woman's peak period of infection with HPV is usually in the first 5-10 years of sexual activity, after which most women have been exposed to and cleared HPV, and developed some immunity against future infection; prevalence across the female population then declines with age. ${ }^{31}$

\section{What about nonavalent vaccination in those previously vaccinated with the quadrivalent or bivalent vaccine?}

Re-vaccination with the nonavalent vaccine in those who have previously received the quadrivalent or bivalent $\mathrm{HPV}$ vaccine is not routinely recommended in Australia or elsewhere..$^{25}$ This is because the major benefit of HPV vaccination is derived from effective protection against the two most oncogenic types: HPV 16 and 18. Additionally, the quadrivalent vaccine provides some cross-protection against HPV 31 and the bivalent vaccine provides a high degree of cross-protection against types 31,33 and $45 .{ }^{32}$ The chance of an individual being persistently infected with any one of the five additional HPV types and developing disease or cancer from them is low. It has been estimated that $50 \%$ of HPV infections that will ever cause cervical cancer have been acquired by age 20 years and $75 \%$ by age 30 years, meaning that the older a woman is at the time of vaccination, the lower her chance of benefitting significantly. ${ }^{33}$ Cervical screening remains the most important additional cervical cancer prevention strategy in sexually active women.

Should a previously vaccinated individual wish to receive a nonavalent 
vaccine course, a trial found a rate of any injection site reaction of $91 \%$, comparable with studies of vaccine-naïve women, although rates of severe swelling were higher (7.6\%). ${ }^{24,34}$ Antibody titres against the additional types are lower than in previously unvaccinated individuals, but the significance of this, if any, is not known. ${ }^{34}$

\section{Key points ${ }^{25}$}

- A two-dose HPV vaccine schedule is now in use for individuals aged 14 years or under at the time of the first dose, with a spacing between doses of 6-12 months (and no closer together than six months). It is applicable to all HPV vaccines (bivalent, quadrivalent and nonavalent).

- Those aged 15 years and above at the time of the first dose, or those with significant immunocompromise, require three doses with a dose spacing of zero, two and six months.

- There is never a need to restart an interrupted HPV vaccine course. Any person who received the first dose at the age of 14 years or under, but who has received no further doses, can receive a second and final dose to complete the course as long as the interval between doses is six months or more. Interrupted three-dose courses should adhere to minimum intervals between dose two and three (three months). In fact, the wider the spacing between doses, the better the immune response.

- A course commenced with one HPV vaccine may be completed with another, noting that protection against any HPV types not covered by each dose may be less complete.

- Catch-up HPV vaccination is now available for those aged up to 19 years. Remember to ask older teens who may have missed out at school. If they are unsure of their HPV vaccination status, you can call the HPV register on 1800 HPV REG (1800 478 734) and obtain their vaccination status on the spot.

- Groups for whom HPV vaccine may be of particular benefit, but for whom it is not nationally funded, include:
- MSM

- those who are significantly immunocompromised

- women post treatment for cervical intraepithelial neoplasia.

- A second course of HPV vaccination with nonavalent vaccine, in those who have received the quadrivalent or bivalent HPV vaccine, is not routinely recommended. This is because the most substantial benefit of HPV vaccination is achieved through protection against the most oncogenic HPV types 16 and 18, which are common to all three vaccines.

- The most important cervical cancer prevention strategy in sexually active women is screening, regardless of their vaccination status.

\section{Useful resources}

- Immunise Australia Program HPV information

- Clinical fact sheet for GPs: https:// beta.health.gov.au/resources/ publications/hpv-vaccine-gardasilr9clinical-advice-fact-sheet-for-gps

- Brochure for parents: https:// beta.health.gov.au/resources/ publications/hpv-vaccinepreventing-human-papillomavirushpv-cancers-and-diseases-by-0

- Video for parents: https://beta.health.gov.au/resources/ videos/hpv-vaccine-preventinghuman-papillomavirus-hpv-cancersand-diseases-by-vaccination

- Immunisation handbook HPV chapter: www.immunise.health.gov.au/internet/ immunise/publishing.nsf/Content/ Handbook10-home handbook10part4 handbook10-4-6

- National Centre for Immunisation Research \& Surveillance frequently asked questions and fact sheets: http://ncirs.edu.au/provider-resources/ ncirs-fact-sheets/

- HPV register (see 'useful links' for infographics summarising nonavalent HPV vaccine for providers and patients): www.hpvregister.org.au

- Cancer Council Australia HPV information site: www.hpvvaccine.org.au

\section{Author}

Julia ML Brotherton B Med (Hons), MPH (Hons), GradDipAppEpi, FAFPHM, PhD, GAICD, Medical Director, Registries and Research, Victorian Cytology Service; Honorary Principal Fellow, School of Population and Global Health, University of Melbourne, Vic. jbrother@vcs.org.au

Competing interests: JMLB has been an investigator on investigator-initiated HPV epidemiology studies that have received unrestricted partial funding for laboratory components from Seqirus (cervical cancer typing study) and MSD (recurrent respiratory papillomatosis study) but has never received any personal financial benefits.

Provenance and peer review: Not commissioned, externally peer reviewed.

\section{References}

1. Schiller J, Lowy D. Explanations for the high potency of HPV prophylactic vaccines. Vaccine 2018. pii: S0264-410X(18)30019-7. doi: 10.1016/j. vaccine.2017.12.079. [Epub ahead of print]

2. Ali $\mathrm{H}$, Donovan $\mathrm{B}$, Wand $\mathrm{H}$, et al. Genital warts in young Australians five years into national human papillomavirus vaccination programme: National surveillance data. BMJ 2013;346:f2032. doi: 10.1136/bmj.f2032.

3. Tabrizi S, Brotherton JML, Kaldor JM, et al. Assessment of herd immunity and cross-protection after a human papillomavirus vaccination programme in Australia: A repeat cross-sectional study. Lancet Infect Dis 2014;14(10):958-66. doi: 10.1016/S1473-3099(14)70841-2.

4. Brotherton JML, Gertig DM, May CL, Chappell GA, Saville M. HPV vaccine impact in Australian women: Ready for an HPV-based screening program. Med J Aust 2016;204(5):184.

5. Gertig DM, Brotherton JML, Budd AC, Drennan K, Chappell G, Saville AM. Impact of a populationbased HPV vaccination program on cervical abnormalities: A data linkage study. BMC Med 2013;11:227. doi:10.1186/1741-7015-11-227.

6. Novakovic D, Cheng ATL, Zurynski Y, et al. A prospective study of the incidence of juvenileonset recurrent respiratory papillomatosis after implementation of a National HPV Vaccination Program. J Infect Dis 2018;217(2):208-12. doi: 10.1093/infdis/jix498.

7. National HPV Vaccination Program Register. HPV vaccination coverage by dose number (Australia) for males/females by age group in mid 2016. National HPV Vaccination Program Register, 2017. Available at www.hpvregister.org.au/research/ coverage-data [Accessed 12 July 2017].

8. Ndiaye C, Mena M, Alemany L, et al. HPV DNA, E6/E7 mRNA, and p16INK4a detection in head and neck cancers: A systematic review and metaanalysis. Lancet Oncol 2014;15(12):1319-31. doi: 10.1016/S1470-2045(14)70471-1.

9. Alemany L, Saunier M, Alvarado-Cabrero l, et al. Human papillomavirus DNA prevalence and type distribution in anal carcinomas worldwide. Int J Cancer 2015;136(1):98-107. doi: 10.1002/ijc.28963.

10. Alemany L, Cubilla A, Halec G, et al. Role of human papillomavirus in penile carcinomas worldwide. Eur Urol 2016;69(5):953-61. doi: 10.1016/j.eururo.2015.12.007.

11. Serrano B, de Sanjosé $S$, Tous $S$, et al. Human papillomavirus genotype attribution for HPVs 6, 11, $16,18,31,33,45,52$ and 58 in female anogenital lesions. Eur J Cancer 2015;51(13):1732-41. doi: 10.1016/j.ejca.2015.06.001. 
12. Li N, Franceschi S, Howell-Jones R, Snijders PJ, Clifford GM. Human papillomavirus type distribution in 30,848 invasive cervical cancers worldwide: Variation by geographical region, histological type and year of publication. Int $J$ Cancer 2011;128(4):927-35. doi: 10.1002/ijc.25396.

13. Serrano B, Alemany $L$, Tous $S$, et al. Potential impact of a nine-valent vaccine in human papillomavirus related cervical disease. Infect Agents Cancer 2012;7(1):38. doi: 10.1186/17509378-7-38.

14. Brotherton JML, Tabrizi SN, Phillips S, et al. Looking beyond human papillomavirus (HPV) genotype 16 and 18: Defining HPV genotype distribution in cervical cancers in Australia prior to vaccination. Int J Cancer 2017;141(8):1576-84. doi: 10.1002/ijc.30871.

15. Luxembourg A, Moeller E. 9-Valent human papillomavirus vaccine: A review of the clinical development program. Expert Rev Vaccines 2017;16(11):1119-39. doi: 10.1080/14760584.2017.1383158.

16. Huh W, Joura E, Giuliano AR, et al. Final efficacy, immunogenicity, and safety analyses of a ninevalent human papillomavirus vaccine in women aged 16-26 years: A randomised, double-blind trial. Lancet 2017;390(10108):2143-59. doi: 10.1016/S0140-6736(17)31821-4.

17. Van Damme P, Meijer CJLM, Kieninger D, et al A phase III clinical study to compare the immunogenicity and safety of the 9-valent and quadrivalent HPV vaccines in men. Vaccine 2016;34(35):4205-12. doi: 10.1016/j. vaccine.2016.06.056.

18. Castellsagué X, Giuliano AR, Goldstone S, et al. Immunogenicity and safety of the 9-valent HPV vaccine in men. Vaccine 2015;33(48):6892-901. doi:10.1016/j.vaccine.2015.06.088.

19. D'Addario M, Redmond S, Scott P, et al. Two-dose schedules for human papillomavirus vaccine: Systematic review and meta-analysis. Vaccine 2017;35(22):2892-901. doi: 10.1016/j. vaccine.2017.03.096.

20. World Health Organization. Human papillomavirus vaccines: WHO position paper, May 2017. Wkly Epidemiol Rec 2017;92(19):241-68.
21. Iversen $\mathrm{OE}$, Miranda MJ, Ulied A, et al. Immunogenicity of the 9-Valent HPV vaccine using 2 -dose regimens in girls and boys vs a 3-dose regimen in women. JAMA 2016;316(22):2411-21. doi: 10.1001/jama.2016.17615.

22. Phillips A, Patel C, Pillsbury A, Brotherton JML, Macartney K. Safety of human papillomavirus vaccines: An updated review. Drug Saf 2017. doi: 10.1007/s40264-017-0625-z. [Epub ahead of print]

23. World Health Organization. Meeting of the Global Advisory Committee on Vaccine Safety, 7-8 June 2017. Wkly Epidemiol Rec 2017;92(28):393-402.

24. Joura $E A$, Giuliano $A R$, Iversen $O E$, et al. $A$ 9-valent HPV vaccine against infection and intraepithelial neoplasia in women. N Engl J Med 2015;372(8):711-23. doi: 10.1056/NEJMoa1405044.

25. Australian Technical Advisory Group on Immunisation (ATAGI). The Australian Immunisation Handbook. 10th edn. Canberra: DoH, updated 2017. Available at www.immunise.health. gov.au/internet/immunise/publishing.nsf/Content/ Handbook10-home [Accessed 17 January 2018].

26. Poynten IM, Tabrizi SN, Jin F, et al. Vaccinepreventable anal human papillomavirus in Australian gay and bisexual men. Papillomavirus Res 2017;3:80-84. doi: 10.1016/j.pvr.2017.02.003.

27. Ingles DJ, Lin HY, Fulp WJ, et al. An analysis of HPV infection incidence and clearance by genotype and age in men: The HPV Infection in Men (HIM) Study. Papillomavirus Res 2015;1:126-35.

28. Department of Health. Human papillomavirus vaccine - Gardasil ${ }^{\circledR}$ 9-Clinical advice for GPs. Fact sheet. Canberra: DoH, 2018. Available at https:// beta.health.gov.au/resources/publications/ hpv-vaccine-gardasilr9-clinical-advice-fact-sheetfor-gps. [Accessed 28 February 2018].

29. Rebolj M, Helmerhorst T, Habbema D, et al. Risk of cervical cancer after completed posttreatment follow-up of cervical intraepithelial neoplasia: Population based cohort study. BMJ 2012;345:e6855. doi: 10.1136/bmj.e6855.

30. Brotherton JML, Wrede CD. Offering HPV vaccination to women treated for high-grade cervical intra-epithelial neoplasia: What do you need to know? Aust N Z J Obstet Gynaecol 2014;54(4):393-94. doi: 10.1111/ajo.12227.
31. Brotherton JML, Condon J, McIntyre PB, et al. Human papillomavirus prevalence to age 60 years among Australian women prevaccination. Sex Health 2015;12(4):353-59. doi: 10.1071/SH15035.

32. Brotherton JML. Confirming cross-protection of bivalent HPV vaccine. Lancet Infect Dis 2017;17(12):1227-28. doi: 10.1016/S14733099(17)30539-X.

33. Burger EA, Kim JJ, Sy S, Castle PE. Age of acquiring causal human papillomavirus (HPV) infections: Leveraging simulation models to explore the natural history of HPV-induced cervical cancer. Clin Infect Dis 2017;65(6):893-99. doi: 10.1093/cid/cix475.

34. Garland SM, Cheung TH, McNeill S, et al. Safety and immunogenicity of a 9-valent HPV vaccine in females 12-26 years of age who previously received the quadrivalent HPV vaccine. Vaccine 2015;33(48):6855-64. doi: 10.1016/j. vaccine.2015.08.059

35. Skinner SR, Apter D, De Carvalho N, et al. Human papillomavirus (HPV)-16/18 AS04-adjuvanted vaccine for the prevention of cervical cancer and HPV-related diseases. Expert Rev Vaccines 2016;15(3):367-87. doi: 10.1586/14760584.2016.1124763.

36. Kavanagh K, Pollock KG, Cuschieri K, et al. Changes in the prevalence of human papillomavirus following a national bivalent human papillomavirus vaccination programme in Scotland: A 7-year cross-sectional study. Lancet Infect Dis 2017;17(12):1293-1302. doi: 10.1016/ S1473-3099(17)30468-1.

37. Brown D, Kjaer SK, Sigurdsson K, et al. The impact of quadrivalent human papillomavirus (HPV; types 6, 11, 16, and 18) L1 virus-like particle vaccine on infection and disease due to oncogenic nonvaccine HPV types in generally HPV-naïve women aged 16-26 years. J Infect Dis 2009;199(7):926-35. doi: 10.1086/597307.

correspondence ajgp@racgp.org.au 